\title{
Síndrome hemolítico urémico atípico
}

Andrés David Villa-García', Camilo Andrés García-Prada² ${ }^{2}$ Mario Alejandro Villabón-González', Sandra Marcela Figueroa-Cárdenas Fernando Iván Meza-Morón ${ }^{4}$

'Servicio de Medicina Crítica y Cuidado Intensivo. Hospital San José. Fundación Universitaria de Ciencias de la Salud, Bogotá DC. Colombia

${ }^{2}$ Servicio de Medicina Interna. Universidad de Antioquia, Medellín. Colombia

${ }^{3}$ Servicio de Urgencias. Universidad del Magdalena. Santa Marta. Colombia.

${ }^{4}$ Servicio de Medicina Crítica y Cuidado Intensivo. Clínica Buenos Aires SAS, Valledupar. Colombia.

Recibido: 15/06/2019

Aceptado: 02/09/2020

En línea: 31/12/2020

Citar como: Villa-García AD, García-Prada CA, Villabón-González MA, Figueroa-Cárdenas SM, Meza-Morón Fl. Síndrome hemolítico urémico atípico. Rev Esp Casos Clin Med Intern (RECCMI). 2020 (Dic); 5(3): 105-108 doi: 10.32818/reccmi.a5n3a2.

Cite this as: Villa-García AD, García-Prada CA, Villabón-González MA, Figueroa-Cárdenas SM, Meza-Morón Fl. Atypical hemolytic uremic syndrome. Rev Esp Casos Clin Med Intern (RECCMI). 2020 (Dec); 5(3): 105-108. doi: 10.32818/reccmi.a5n3a2.

Autor para correspondencia: Camilo Andrés García-Prada. cgarciap4@unicartagena.edu.co

\section{Palabras clave \\ $\triangleright$ Síndrome hemolítico urémico \\ $\triangleright$ Eculizumab \\ $\triangleright$ Plasmaféresis \\ $\triangleright$ Microangiopatía trombótica}

\begin{tabular}{l} 
Keywords \\
\hline$\triangleright$ Uremic syndrome \\
$\triangleright$ Eculizumab \\
$\triangleright$ Plasmapheresis \\
$\triangleright$ Thrombotic microangiopathy
\end{tabular}

\begin{abstract}
Resumen
El síndrome hemolítico urémico atípico es una enfermedad muy rara, de progresión rápida y de alta morbimortalidad, especialmente cuando no se reconoce ni se trata. Se clasifica en formas primaria y secundaria, caracterizada por la tríada de anemia hemolítica microangiopática, trombocitopenia e insuficiencia renal. Presentamos un caso en el puerperio, con manifestaciones clínicas extrarrenales especialmente de origen neurológico y antecedentes de embarazo no controlado. Se descartan otros diagnósticos diferenciales y se inicia manejo con plasmaféresis y eculizumab, lográndose mejoría clínica, analítica y de imagen.

Abstract

Atypical Hemolytic Uremic Syndrome is a very rare, rapidly progressive, high morbidity and mortality illness, especially when it is not recognized and treated. This subtype is classified in primary and secondary. It is characterized by the triad of microangiopathic haemolytic anemia, thrombocytopenia, and renal failure; however, this clinical case occurring mainly with extrarenal clinical manifestations, especially of neurological origin and an uncontrolled pregnancy recent history; other differential diagnoses were excluded. Furthermore were started medical treatment with plasmapheresis and eculizumab, achieving clinical radiological, and blood test improvement.
\end{abstract}

Puntos destacados

$\triangleright$ El síndrome hemolítico urémico atípico constituye una entidad multifactorial.

$\triangleright$ La asociación entre los mecanismos de microangiopatía trombótica en puerperio y el embarazo con el compromiso endotelial multiorgánico del SHUa motiva la sospecha diagnóstica para establecer una estrategia terapéutica temprana y reducir la mortalidad.

\section{Introducción}

Las microangiopatías trombóticas (MAT) se han descrito inicialmente en 1952 por Symners, clasificadas frecuentemente como púrpura trombocitopénica (PTT) y síndrome hemolítico urémico (SHU). El síndrome hemolítico urémico atípico (SHUa) se considera una enfermedad rara con una prevalencia de 3,3 pacientes/millón de habitantes, en la que entre el 9-15\% de los pacientes fallecen en el primer año; un 33\% quedan con secuelas renales y hasta el 63\% de los pacientes pueden presentar afectación en otros órganos distintos al riñón, como manifestaciones neurológicas, gastrointestinales y cardiovasculares'.
A continuación, se presenta un caso clínico, que debuta con fracaso multiorgánico, con excelente respuesta al uso de plasmaféresis y anticuerpos monoclonales.

\section{Caso clínico}

\section{Antecedentes}

Mujer de 20 años de edad con antecedente de síndrome epiléptico como secuela de intoxicación por plaguicidas.

\section{Enfermedad actual}

Trasladada desde un hospital local con historia de 4 días de cefalea global intensa, fiebre y deterioro sensorial, tras puerperio quirúrgico tardío de un embarazo con pobre control. La convulsión generalizada se controla con benzodiacepinas e intubación orotraqueal. 


\section{Exploración física}

En su admisión a la unidad de cuidados intensivos (UCI) presenta presión sanguínea de $80 / 60 \mathrm{mmHg}$, requiriendo soporte vasopresor. Su frecuencia cardíaca es de 103 latidos/minutos, bajo ventilación mecánica y sedoanalgesia (RASS: -3); parámetros ventilatorios dinámicos; reflejos de tallo incompletos; miosis, con gingivo-glosorragia, arreflexia, sin signos piramidales o meníngeos.

\section{Pruebas complementarias}

El hemograma de ingreso documenta bicitopenia y el resto de pruebas analíticas se muestran en la Tabla 1.

Al descartarse neuroinfección se suspende la antibioticoterapia (piperacilinatazobactam + ceftriaxona). Evoluciona con hipersecreción traqueal hemoptoica con cultivo de secreción negativo mostrando datos radiológicos compati-

\begin{tabular}{|c|c|c|c|c|c|c|c|}
\hline Citoquímico de orina & Aspecto & $\mathrm{pH}$ & Densidad & Proteinuria & Eritrocitos & Leucocitos & Proteinuria \\
\hline & Turbio & 7,3 & 1,03 & $20 \times$ campo & 50-60 x campo & $25-30 \times$ campo & 124 mg/día \\
\hline \multirow[t]{2}{*}{ Gases arteriales } & $\mathrm{pH}$ & $\mathrm{PCO}_{2}$ & $\mathrm{PO}_{2}$ & Proteinuria & BE- & PAFI & Lactato \\
\hline & 7,35 & $45 \mathrm{mmHg}$ & $72 \mathrm{mmHg}$ & $15 \mathrm{mmol} / \mathrm{L}$ & $-12,4$ & 213 & $1,3 \mathrm{mmol} / \mathrm{L}$ \\
\hline \multirow[t]{4}{*}{ Perfil renal y metabólico } & Creatinina & BUN & Sodio & Cloro & Potasio & Calcio sérico & Urea \\
\hline & $0,99 \mathrm{mg} / \mathrm{dL}$ & $33,8 \mathrm{mg} / \mathrm{dL}$ & $152,2 \mathrm{mEq} / \mathrm{L}$ & $123 \mathrm{mEq} / \mathrm{L}$ & $4,89 \mathrm{mEq} / \mathrm{L}$ & $9,3 \mathrm{mg} / \mathrm{dL}$ & $29,9 \mathrm{mg} / \mathrm{dL}$ \\
\hline & $\begin{array}{l}\text { Proteínas en } \\
\text { orina de } 24 \mathrm{~h}\end{array}$ & $\begin{array}{l}\text { Depuración de } \\
\text { creatinina en } 24 \mathrm{~h}\end{array}$ & Magnesio & Glicemia & Ácido fólico & Ferritina & Vitamina B12 \\
\hline & 124 mg/día & $112,2 \mathrm{~mL} / 24 \mathrm{~h}$ & $1,56 \mathrm{mg} / \mathrm{dL}$ & $126 \mathrm{mg} / \mathrm{dL}$ & $14,2 \mathrm{mg} / \mathrm{mL}$ & $393 \mathrm{ng} / \mathrm{mL}$ & $288 \mathrm{pg} / \mathrm{m}$ \\
\hline \multirow[t]{8}{*}{ Perfil hematológico } & Hemoglobina & Hematocrito & Leucocitos & Neutrófilos & Linfocitos & Plaqueta & $\begin{array}{l}\text { Reticulocitos } \\
\text { corregidos }\end{array}$ \\
\hline & $7,2 \mathrm{~g} / \mathrm{dL}$ & $18,40 \%$ & $6800 \mathrm{~mm}^{3}$ & $5400 \mathrm{~mm}^{3}$ & $1100 \mathrm{~mm}^{3}$ & $70100 \mathrm{~mm}^{3}$ & 0,66 \\
\hline & ESP & LDH & Haptoglobina & $\begin{array}{l}\text { Actividad } \\
\text { ADAMTS } 13\end{array}$ & TPT & PT & $\begin{array}{l}\text { Control de } \\
\text { PTT }\end{array}$ \\
\hline & Esquistocitos ++ & $1420 \mathrm{u} / \mathrm{L}$ & $240 \mathrm{mg} / \mathrm{dL}$ & $78,40 \%$ & $38,7 \mathrm{~s}$ & $18,7 \mathrm{~s}$ & 30,5 \\
\hline & INR & Fibrinogeno & & & & & \\
\hline & 1,2 & $276 \mathrm{mg} / \mathrm{dL}$ & & & & & \\
\hline & BI & BD & BT & ALT & AST & FA & \\
\hline & $0,36 \mathrm{mg} / \mathrm{dL}$ & $0,85 \mathrm{mg} / \mathrm{dL}$ & $1,21 \mathrm{mg} / \mathrm{dL}$ & $419 \mathrm{U} / \mathrm{L}$ & $482 \mathrm{U} / \mathrm{L}$ & $283 \mathrm{U} / \mathrm{L}$ & \\
\hline \multirow[t]{2}{*}{ Perfil inmunológico } & C3 & C4 & ANA & ANTI DNA & ANCAS & ANTI-SM & Coombs directo \\
\hline & $57 \mathrm{mg} / \mathrm{dL}$ & $18,9 \mathrm{mg} / \mathrm{dL}$ & Negativo & Negativo & Negativo & Negativo & Negativo \\
\hline \multicolumn{8}{|c|}{ Otros estudios } \\
\hline \multicolumn{3}{|l|}{ Punción Lumbar } & \multicolumn{5}{|c|}{$\begin{array}{l}\text { Citoquímico y citológico, VDRL latex para Criptococo, PCR para Herpes virus, } \\
\text { Citomegalovirus, Epstein Barr virus y enterovirus en LCR : no detectable } \\
\text { Positivo E. Coli BLEE (-) }\end{array}$} \\
\hline \multicolumn{3}{|c|}{$\begin{array}{l}\text { Hemocultivos, cultivo de secreción traqueal y urocultivo: } \\
\text { Ecocardiograma }\end{array}$} & \multicolumn{5}{|c|}{$\begin{array}{l}\text { 07/06/2019: FEVI 38\% con hipoquinesia global leve } \\
\text { 24/07/2019: FEVI 72\% sin trastorno de la contractilidad }\end{array}$} \\
\hline VDRL: No reactivo & VIH: Negativo & IgM Dengue: Negat & Ag S Hepa & tis B: Negativo & Hepatitis C: $\mathrm{Ne}$ & gativo & \\
\hline \multicolumn{8}{|c|}{ 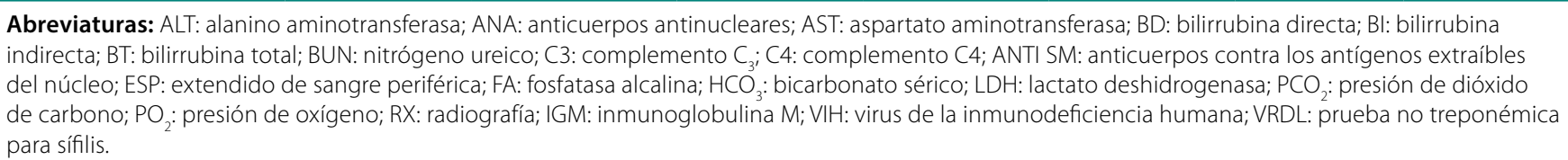 } \\
\hline
\end{tabular}

Tabla 1. Evolución de pruebas analíticas.

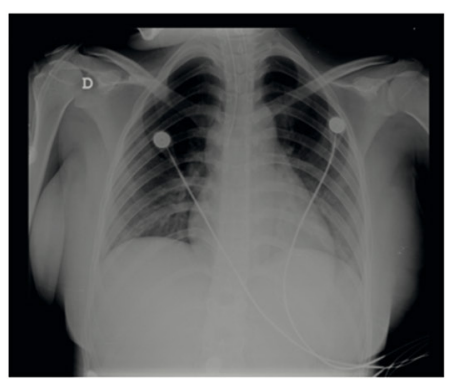

$13 / 06 / 19$ A

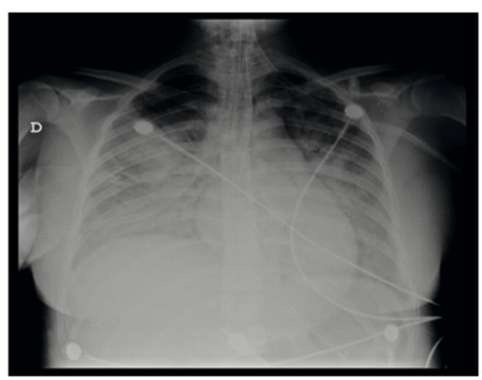

$15 / 06 / 19$ B

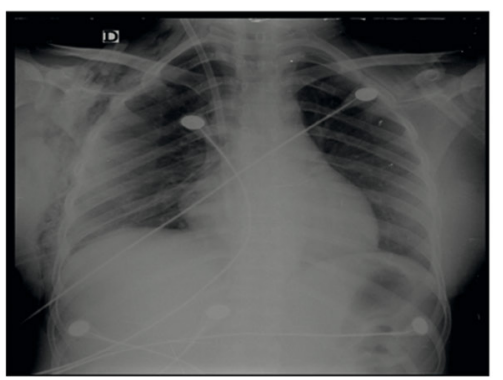

$22 / 06 / 19 \mathrm{C}$

Figura 1. Múltiples opacidades reticulares parahiliares bilateral, silueta cardíaca aumentada de tamaño, compatible con edema agudo de pulmón. 
bles con edema agudo de pulmón (Figura 1). Se constata miocardiopatía en ecocardiograma con FEVI de 38\%, hipoquinesia global leve, sin valvulopatía.

La resonancia magnética (RM) simple cerebral muestra en la región parietal derecha lesión extraaxial (subdural), laminar aproximadamente de $3 \mathrm{~mm}$ de espesor con señal hiperintensa en T1, T2; flair con mínimo efecto de masa y pequeñas señales nodulares y puntiformes hiperintensas en $T 2$ y flair en la sustancia blanca frontoparietal izquierda y pedículo cerebeloso medio homolateral sin efecto de masa ni restricción en la secuencia de difusión (Figura 2A). Se describe control de resonancia magnética posterior a primera dosis de eculizumab que visualiza pequeños focos de gliosis supra e infratentorial, sin hematomas, sistema ventricular sin alteraciones ni desplazamientos (Figura 2B).

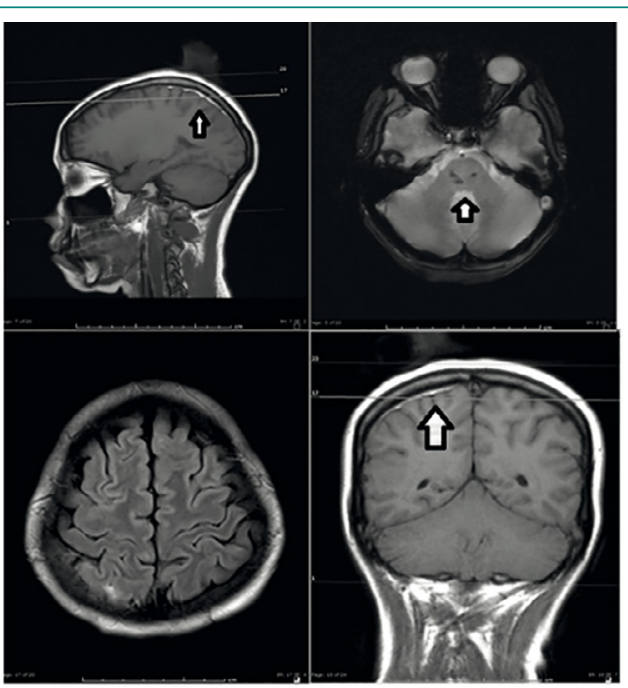

$24 / 06 / 17 \AA$

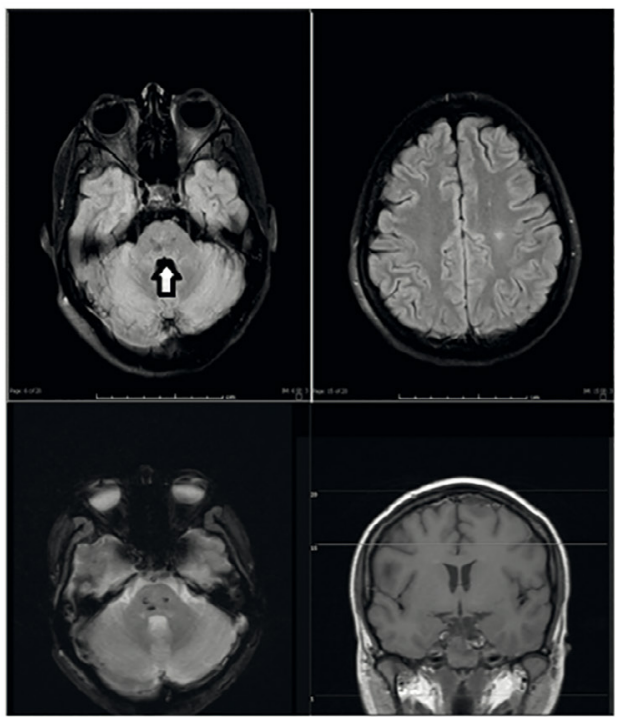

$15 / 07 / 19 B$

Figura 2. Resonancia magnética craneal.

\section{Evolución}

Se inicia terapia soporte con esteroides (metilprednisolona intravenoso $5 \mathrm{mg} / \mathrm{kg} / \mathrm{día}$ seguido de oral durante 7 días) y hemotransfusión, con mejoría discreta de trombocitopenia y anemia. En consenso con el Servicio de Nefrología se programa plasma- féresis, con restablecimiento de parámetros paraclínicos y radiográficos, disminución de opacidades lográndose extubación al décimo día con déficit neurológico parcial, reactividad pupilar preservada, disquinesias cervicales, hiporreflexia en miembros superiores tono y trofismo normal, sensibilidad nociceptiva conservada sin signos de irritación meníngea con electroencefalograma que concluye lentificación de la actividad de fondo, sugestivo de encefalopatía.

Los estudios complementarios para estudio de microangiopatías trombóticas (MAT) secundaria (anticuerpos antifosfolípido y anticoagulante lúpico) fueron negativos, toxina Shiga negativa sin clínica digestiva ni compromiso renal con afectación neurológica, hematológica, pulmonar y cardíaca renal que, sumado a la actividad regular de ADAMTS 13, se admite como sospecha SHUa indicando eculizumab (900 mg por perfusión intravenosa semanal durante 4 semanas) previa inmunización para meningococo e influenza. La evolución neurológica fue favorable, con RM cerebral sin hematomas y además recuperación de disfunción miocárdica.

\section{Diagnóstico}

Síndrome hemolítico urémico atípico.

\section{Discusión y conclusión}

Las microangiopatías trombóticas (MAT) son un grupo de entidades distintas, caracterizadas por lesión endotelial y formación de trombos en pequeños vasos sanguíneos. Esta limitación del flujo en la microvasculatura, puede ocasionar isquemia aguda siendo el riñón el órgano más afectado (80\%)ํ.

El compromiso extrarrenal es relativamente frecuente en el SHUa, entre el 10 y el $30 \%$, predominando el compromiso cerebral (48\%), más frecuente en la PTT que el SHUa ${ }^{1,2}$. También se describe insuficiencia cardíaca, miocardiopatía, vasculopatía isquémica periférica y, con menor frecuencia, coronariopatía como afectación cardíaca y manifestaciones gastrointestinales hasta un fracaso multiorgánico'.

Esta inespecificidad en nuestra paciente dificulta el diagnóstico diferencial con otras causas de MAT, convirtiéndose en una enfermedad con comportamiento progresivo potencialmente mortal ${ }^{2}$. La PTT y el SHU como subgrupo representan un reto diagnóstico y terapéutico por su curso clínico heterogéneo caracterizándose por hemólisis con LDH elevada, haptoglobina casi indetectable, trombocitopenia, esquistocitos en sangre periférica y lesión orgánica o sistémica ${ }^{1,2}$.

Se reconoce el origen multifactorial de las MAT. En algunos individuos existe predisposición genética activada por factores ambientales, fármacos, infecciones, trastornos autoinmunes y traumatismos ${ }^{3}$. En el SHUa, la vía alterna del complemento siempre está activa, pudiendo auto-amplificar su respuesta bajo estas condiciones, causando un desequilibrio entre los mecanismos reguladores (normalmente disminuidos) y excitadores, predisponiendo a efectos crónicos nocivos a nivel tisular y celular ${ }^{3,4}$ explicando la variedad de manifestaciones clínicas que repercuten en una tasa alta de morbimortalidad y enfermedad crónica².

La trombosis intravascular en la PTT es consecuencia de una deficiencia de la actividad metaloproteasa de ADAMTS13 (A Desintegrin and Metalloproteninasa with ThromboSpondin type 1 motif, member 13) ${ }^{5}$, encargada de fragmentar los multímeros largos del factor de Von Willebrand bloqueada por anticuerpos circulantes de lgG liberados por causas genéticas o adquiridas'.

Nuestra paciente presenta bicitopenia, Coombs directo negativo, LDH elevada y esquistocitos, sin hiperazoemia ni proteinuria en rango nefrótico, aunque con hematuria, obligando a excluir uroinfección por urocultivo, considerándose la MAT como diagnóstico diferencial, indicando medidas de sostén en las primeras 24-48 horas. 
Algunas patologías hipertensivas del embarazo se relacionan con mecanismos de microangiopatía trombótica como el síndrome HELLP y antifosfolípido imitando clínica de SHUa o PTT como causas de MAT secundarias. En una serie de casos, pacientes con síndrome HELLP presentaron mutaciones y desregulación del complemento, estableciendo como hipótesis una condición comparable coexistente en nuestra paciente, prevalente hasta en el 70\% de casos de MAT, asociada al embarazo, no lográndose concluir por datos prenatales objetivos insuficientes ${ }^{2,3,6}$

La mortalidad elevada en las primeras 48 horas en pacientes graves se debe a disfunción multiorgánica. La sepsis se presenta concomitante como en nuestro caso, empeorando el pronóstico, sometiendo a discusión el desarrollo de infección como epifenómeno de MAT o su presentación concurrente, motivando el tratamiento precoz!

La transfusión de hemoderivados produjo mejoría; sin embargo, la plasmaféresis determinó mayor descenso en la LDH (Figura 3). Su inicio en las primeras 24 horas de debut clínico reduce la mortalidad y las secuelas clínicas².

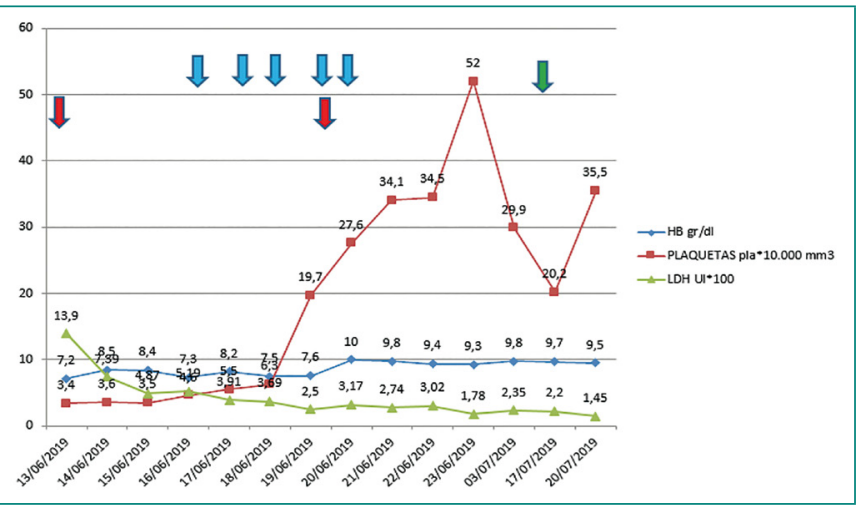

Figura 3. Evolución de los parámetros de laboratorio durante el tratamiento: recambios plasmáticos, 5 sesiones (flecha azules)

transfusiones, 2 (flecha roja). Además, se inició eculizumab el 17 de julio de 2019 (flecha verde). Los valores de LDH se encuentran en escala de 1×102 y los valores de plaquetas se encuentran en escala de $1 \times 104$.
La plasmaféresis pasa a terapia alternativa, con la introducción del eculizumab y su aprobación por la FDA en 2011 como tratamiento de primera elección en SHUa al inhibir la conversión de C5 a C5a; corrige las alteraciones hematológicas, manifestaciones severas renal y extrarrenales, e incluso logra una remisión completa de la enfermedad. Su uso evidenció una respuesta neurológica en la función motor-sensitiva con la primera dosis anticipando la posibilidad de sobrevida y reversibilidad de disfunciones orgánicas ${ }^{4}$.

\section{Bibliografía}

1. Córdoba JP, Contreras KM, Larrarte C, Espitaleta Z, González LE, Ibarra M, et al. Síndrome hemolítico urémico atípico, revisión de la literatura y documento de consenso. Enfoque diagnóstico y tratamiento. Rev. Colomb. Nefrol. 2015; 2(1): 19-0. Disponible en: https://revistanefrologia.org/index php/rcn/article/view/199.

2. Camargo Assis F, Ortiz Ruiz G, Garay Fernández M, Córdoba JP, Yepes D, González M, et al. Microangiopatías trombóticas en la unidad de cuidados intensivos. Acta Colombiana de Cuidado Intensivo. 2017; 17(2): 117-128. Doi. org/10.1016/j.acci.2017.02.003

3. Huerta A, Arjona E, Portoles J, Lopez-Sanchez P, Rabasco C, Espinosa M, et al, A retrospective study of pregnancy-associated atypical hemolytic uremic syndrome. Kidney Int. 2018; 93(2): 450-459. doi: 10.1016/j.kint.2017.06.022.

4. Fakhouri F, Fila M, Provôt F, Delmas Y, Barbet C, Châtelet V, et al. Pathogenic Variants in Complement Genes and Risk of Atypical Hemolytic Uremic Syndrome Relapse after Eculizumab Discontinuation. Clin J Am Soc Nephrol. 2017; 12(1): 50-59. doi: 10.2215/CJN.06440616.

5. Campistol JM, Arias M, Ariceta G, Blasco M, Espinosa L, Espinosa M, et al. An update for atypical haemolytic uraemic syndrome: diagnosis and treatment. A consensus document. Nefrologia. 2015;35(5):421-47. doi: 10.1016/j.nefro.2015.07.005.

6. Müller-Calleja N, Ritter S, Hollerbach A, Falter T, Lackner KJ, Ruf W. Complement $\mathrm{C} 5$ but not $\mathrm{C} 3$ is expendable for tissue factor activation by cofactor-independent antiphospholipid antibodies. Blood Adv. 2018; 2(9): 979-986. doi: 10.1182/bloodadvances.2018017095. 